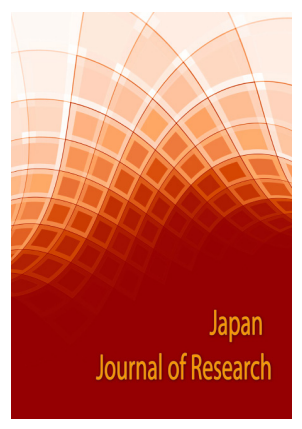

Correspondence

Marinova L.

Oncology Center, Russe, Bulgaria

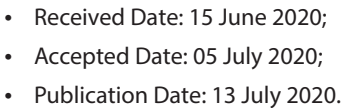

Copyright

(c) 2020 Science Excel. This is an openaccess article distributed under the terms of the Creative Commons Attribution 4.0 International license.

\title{
Comparative dosimetric analysis of the left breast carcinoma radiotherapy irradiation techniques with deep inspiration breathing and free breathing
}

\author{
Sp. Kovacheva-Damyanova', K. Zhelev' MD, Z. Zahariev' MD, R. Krasteva' MD, \\ L. Marinova² PhD \\ 'Oncology Center, MBAL Uni Hospital, Panagiurishte, Bulgaria \\ ${ }^{2}$ Oncology Center, Russe, Bulgaria
}

\begin{abstract}
Breast cancer $(\mathrm{BC})$ is the second most common malignancy in the world, most commonly diagnosed in women. Radiotherapy (RT) for left breast cancer (LBC) is followed by a higher incidence of ischemic heart disease and an increased risk of death.

Radiotherapy was applied for 76 patients with LBC using tangential 3D conformal RT (3D CRT) with two different radiotherapy techniques - free breathing (FB) and deep inspiration breath-hold (DIBH). The patients were treated in the period 2016 - 2019 in the Oncology Center, Uni Hospital, Panagyurishte, Bulgaria.

We present a comparative dosimetric analysis of the mean heart dose (MHD), mean and maximum left anterior descending (LAD) coronary artery doses and mean and maximum left ventricle doses reported with two different radiotherapy techniques $\mathrm{FB}$ and $\mathrm{DIBH}$. The linear regression statistical analysis shows a significant decrease in the MHD with the DIBH technique. The left breast cancer RT with the DIBH technique significantly reduces the mean heart dose and allows controlling the maximum $L A D$ dose and the maximum ventricle dose.
\end{abstract}

\section{Introduction}

Breast cancer $(\mathrm{BC})$ is the second most common malignant tumour in the world and the most common cancer among women (1). During 2018 the cases of newly diagnosed with BC were around 2.1 million, $24.2 \%$ of the newly diagnosed cancer women, or $11.6 \%$ of all new patients. BC is the fifth reason for death in women, i.e. 627 000 deaths for 2018 or $15 \%$ of all reasons for death (2). The prognosis of newly diagnosed BC is overall good in the economically well developed countries (2). Adjuvant radiotherapy (RT) in left breast cancer (LBC) was followed by a higher incidence of ischemic heart disease (IHD) and an increased risk of premature cardiac death $(3,4)$. Comparing to the right breast cancer, the reason is a higher radiation doses to the heart and coronary arteries, especially during respiratory movements of the chest (5). In order to optimize target coverage while maintaining a low dose to organs at risk (OAR), the RT techniques with respiratory gating $(\mathrm{RG})$, such as enhanced inspiration gating and deep-inspiration breath hold (DIBH), have successfully been implemented in RT centres during the last decade (6-9). Irradiation during deep inspiration breath-hold (DIBH) increases the distance between planning target volume (PTV) and heart $(10,11)$. In this study, we present a comparative dosimetric analysis of the radiation doses to the heart, left anterior descending (LAD) coronary artery (left atrial) and left ventricle.

\section{Material and Methods}

This study is quantitative retrospective dosimetric analysis of postoperative RT in 76 patients with left breast cancer after breast conserving surgery between 2016-2019 in the Oncology Center MBAL Uni Hospital, Panagiurishte, Bulgaria. 58 patients (76.31\%) were histologically verified with invasive ductal carcinoma and 18 (23.69\%) - invasive lobular carcinoma. 42 patients $(51 \%)$ were stage I, 28 (36.84\%) - stage II, 1 (1.31\%) - stage III. Median age was 49 (range: 22-79). Radiotherapy was applied for 76 patients with LBC using tangential fields $3 \mathrm{D}$ conformal $\mathrm{RT}$ technique during $\mathrm{DIBH}$ and free breathing (FB).

All patients underwent RT of left breast, 50 (65.8\%) had regional lymph node included. Adjuvant chemotherapy was applied to $74(97.37 \%)$ patients. CT simulation with DIBH was performed on 43 patients (57\%) with RPM Gating Varian Medical System, whereas 33 patients (43\%) were scanned and irradiated during free breathing (FB).

Dosimetric planning was performed on Eclipse Planning system with algorithm AAA13.6. Radiation treatment was performed on linear accelerator True Beam Edge with tangencial fields 3D CRT technique to total dose (TD) 50 Gy in 25 fractions for 5 weeks. The goal was $95 \%$ of the dose to be delivered to the planned target volume (PTV) and in the same time maximum organs at risk (OAR) spearing to be achieved. 


\section{Dosimeric results}

This comparative dosimetric analysis examines the mean and maximum doses to the heart and its structures (mean heart dose (MHD), mean and maximum left anterior descending (LAD) coronary artery doses and maximum left ventricle doses) for two different radiotherapy techniques FB and DIBH. All dosimetric plans were calculated for tangencial fields 3D CRT technique. The goal was optimal homogeneity to the PTV and maximum OAR spearing. Mean dosimetric coverage for 95\% isodose (V95\%) for the DIBH group was $91.71 \%$ [Figure 1], whereas for the FB group - 91.3\% [Figure 2]. Mean dose of the PTV was $100.79 \%$ for the DIBH group, whereas
$100.26 \%$ for the FB group. Mean maximum dose for the DIBH group was $110.12 \%$, whereas $110.54 \%$ for the FB group. Figure 1 and Figure 2 represent axial slice of dose distribution in PTV for patients. Yellow and pink arrows show the lateral heart-to-chest distance (HCD yellow) and the cardiac contact distance (CCD - pink), which are individual for each patient.

The relation between the heart and its structures was examined in case of DIBH and FB techniques. All patients were grouped by intervals in order to divide them into subgroups according to their mean heart dose, mean LAD dose, max LAD dose, mean and max ventricle doses [Figures 3,4,5,6,7]

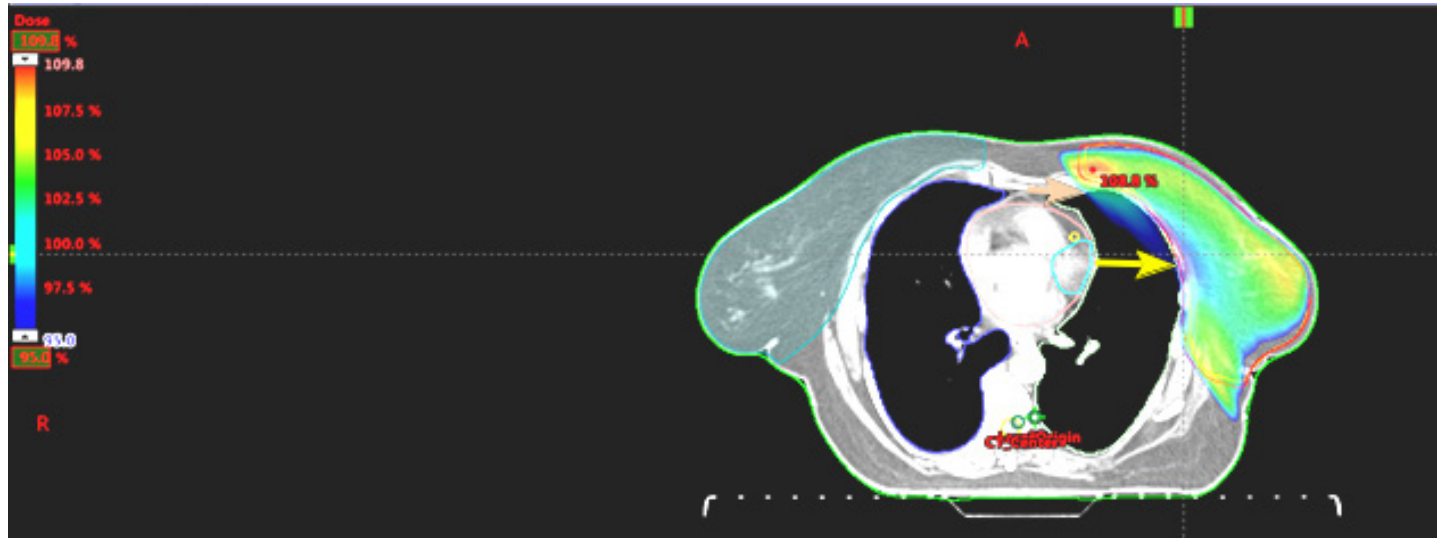

Figure 1. PTV dose distribution (V95\%) in DIBH technique group with HCD and CCD parameters.

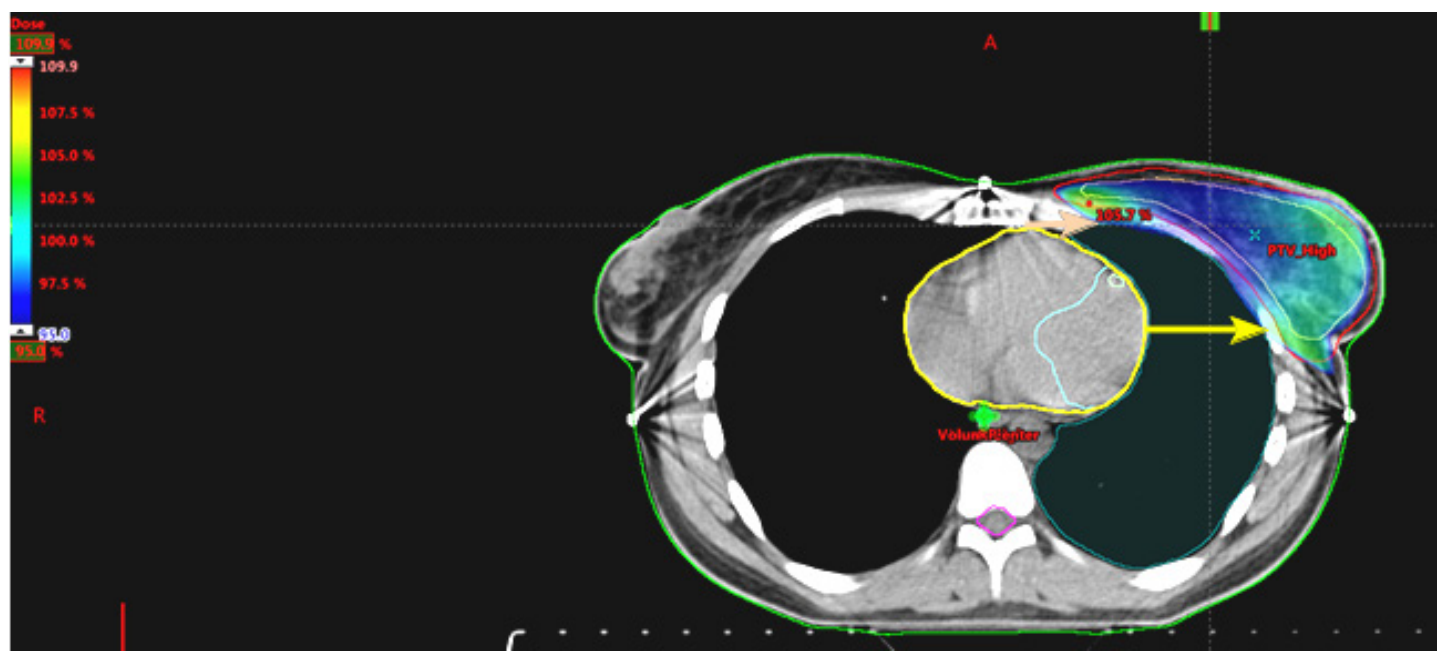

Figure 2. PTV dose distribution (V95\%) in the free breathing (FB) group with HCD and CCD parameters.

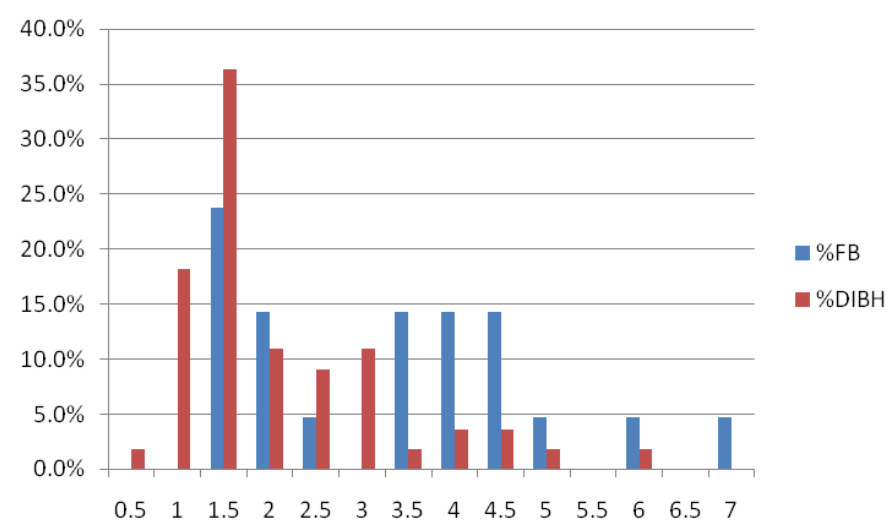

Figure 3. Distribution of patients (\%) by subgroups compared to mean heart dose (Gy).

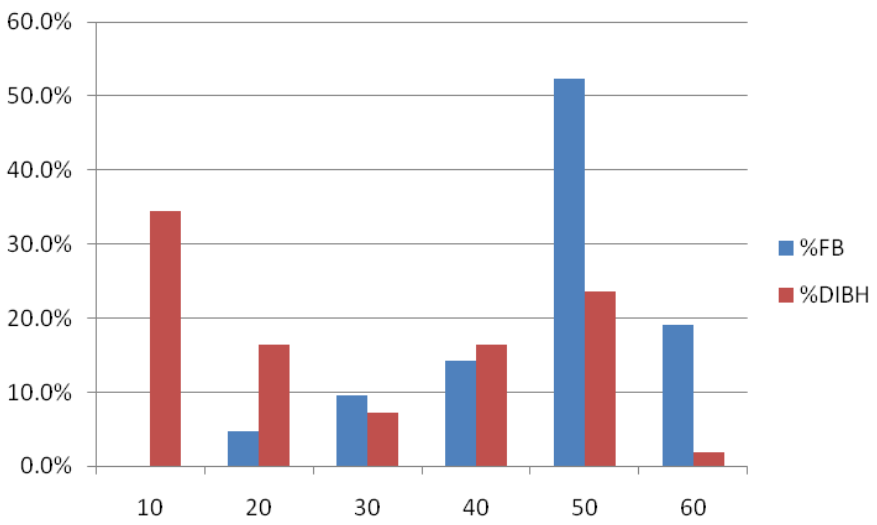

Figure 4. Distribution of patients (\%) by subgroups compared to max LAD dose (Gy). 


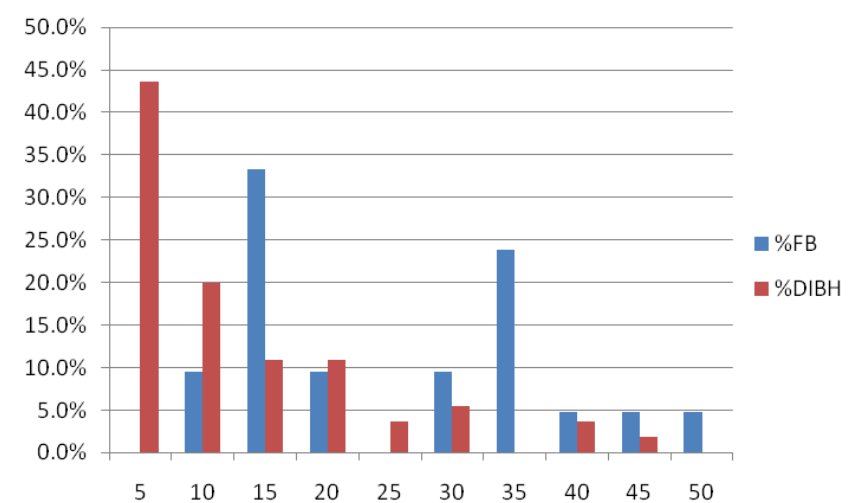

Figure 5. Distribution of patients (\%) by subgroups compared to mean LAD dose (Gy).

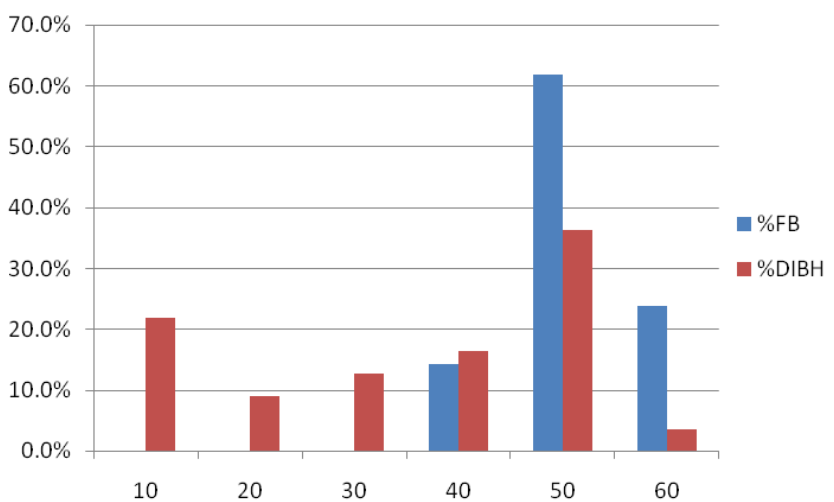

Figure 6. Distribution of patients (\%) by subgroups compared to max ventricle dose (Gy).

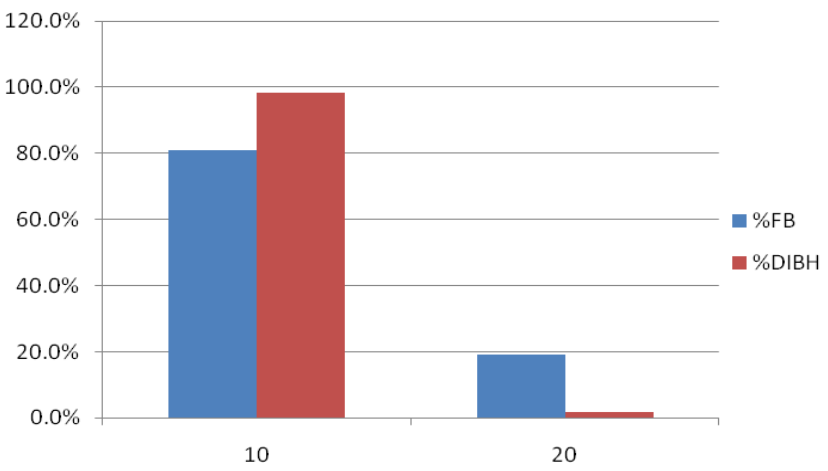

Figure 7. Distribution of patients (\%) by subgroups compared to mean ventricle dose (Gy)

The mean heart dose (MHD) is $1.58 \pm 1.13 \mathrm{~Gy}$ in DIBH group, and $3.09 \mathrm{~Gy} \pm 1.86 \mathrm{~Gy}$ in $\mathrm{FB}$ group. The mean LAD dose is $10.56 \pm$ $10.21 \mathrm{~Gy}$ in DIBH group, and $22.62 \pm 11.87 \mathrm{~Gy}$ in FB group. The mean maximum LAD dose is $23.65 \pm 16.89$ Gy in DIBH group and 42.16 $\pm 10.29 \mathrm{~Gy}$ in FB group. The mean ventricle dose is $3.27 \pm 2.62 \mathrm{~Gy}$ in DIBH group, and $6.98 \pm 3.91 \mathrm{~Gy}$ in FB group. The mean maximum ventricle dose is $30.14 \pm 16.81 \mathrm{~Gy}$ in DIBH group, and $46.98 \pm 5.35 \mathrm{~Gy}$ in FB group. Irradiation with free breathing technique shows two times higher values for mean heart dose, mean ventricle and mean LAD dose. The mean maximum LAD dose is increased by $56 \%$, and maximum ventricle dose by $65 \%$. Analysis of the relation between all those values and confirmation of linear correlation between the two techniques is performed by calculating correlation coefficient $(r)$ and linear regression model with determination coefficient (R2) (Figure 8).

\author{
$R^{2}=\frac{\text { SSR }}{\text { SST }}$ \\ SSR-Sum of Squared Regression \\ SST- Total Sum of Squares \\ $\mathbf{R}^{2}$ - coefficient of determination (regression) - $\mathbf{R}^{2}$ values varies between 0 and 1 ; The linear \\ connection between the two factors is greater when the values are closer to 1.
}

Figure 8. Determination coefficient formula, linear regression model.

The analysis of DIBH radiation technique shows correlation between the values of mean heart dose and maximum $\mathrm{LAD}$ dose $/ \mathrm{R}^{2}=0.5362$, $\mathrm{r}=0.349$. This linear relation is not confirmed when $\mathrm{FB}$ techniques is applied / $\mathrm{R}^{2}=0.1216, \mathrm{r}=0.349$ [Figure 9].
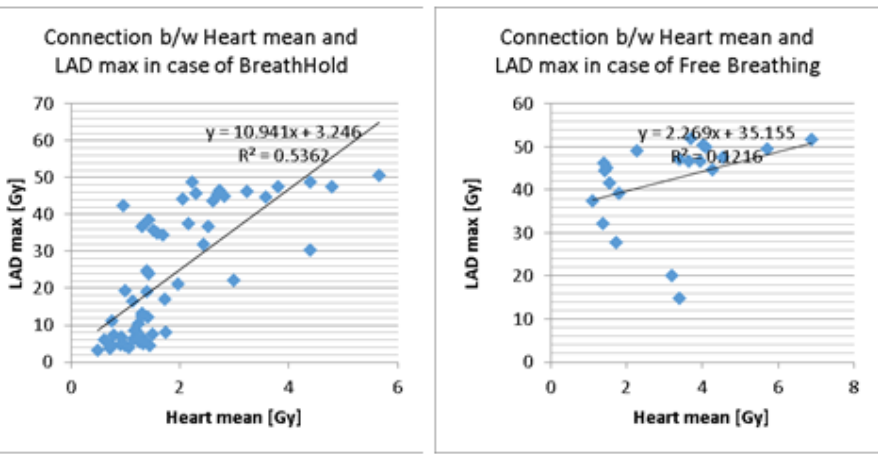

Figure 9. Relation between mean heart dose and maximum dose in LAD with both techniques - DIBH and FB.

The analysis of the DIBH group indicates dependence between mean heart dose and mean LAD dose $\mathrm{R}^{2}=0.751, \mathrm{r}=0.867$, which is confirmed in the FB group $\mathrm{R}^{2}=0.7197, \mathrm{r}=0.848$ [Figure 10].
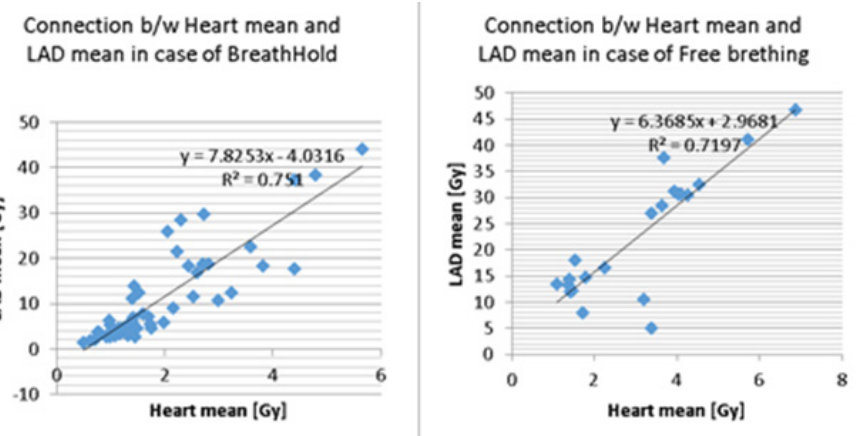

Figure 10. Relation between mean heart dose and mean LAD dose with both techniques - DIBH and FB.
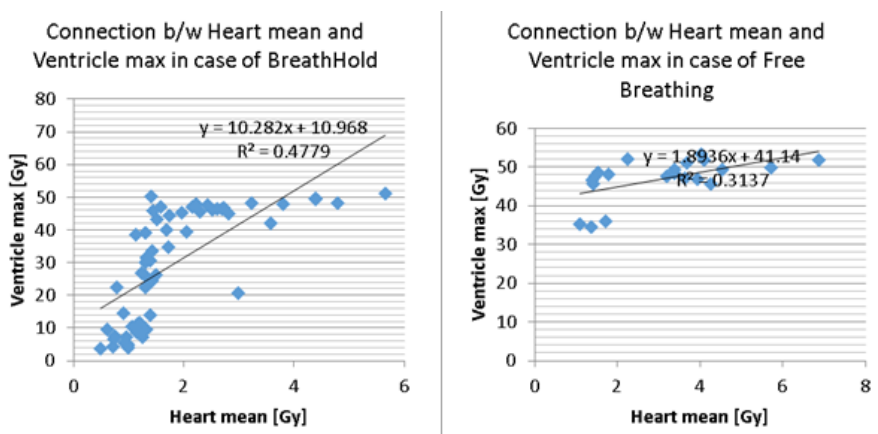

Figure 11. The relation between the mean heart dose and the maximum left ventricle dose with both techniques - DIBH and FB. 
The analysis of DIBH radiation technique shows correlation between the values of mean heart dose and maximum ventricle dose $/ \mathrm{R}^{2}=$ $0.4779, \mathrm{r}=0.691$, the corresponding correlation is not maintained by the $\mathrm{FB}$ group $/ \mathrm{R}^{2}=0.0495, \mathrm{r}=0.560$ [Figure 11].

The analysis of the DIBH technique shows a relation between the values of mean heart dose and mean left ventricle dose / $R^{2}=0.7774$, $\mathrm{r}=0.882$, the corresponding is confirmed in the group with $\mathrm{FB} / \mathrm{R}^{2}=$ $0.9076, r=0.953$ [Figure 12].
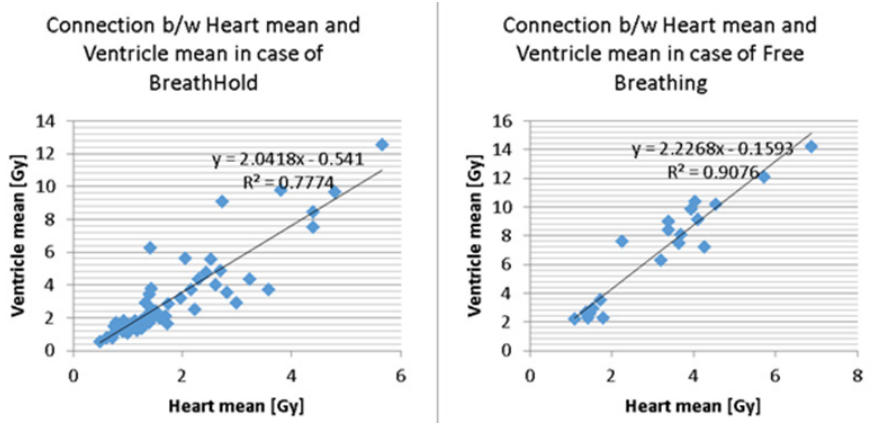

Figure 12. The relation between the mean heart dose and the maximum left ventricle dose with both techniques - DIBH and FB.

\section{Discussion}

Since the beginning of the application of 3D CRT for breast cancer irradiation it proved to be a challenge to radiation professionals. The maximum heart dose has been compared to irradiation with various static 3D conformal techniques- with separate tangential fields and with tangential fields with one separate field for the regional lymph nodes irradiation. The maximum dose values to the heart are directly dependent on the applied radiation technique. In the left breast RT with tangential fields 3D CRT, the maximum dose to the heart is 20-45 Gy, and with tangential fields in combination with a separate additional field- 0-30 Gy (12). The optimization of the breast cancer $3 \mathrm{D}$ conformal $\mathrm{RT}$, continues by standardizing various radiation techniques in order to reduce the dose to the heart and lung (13).

Affecting the left anterior descending (LAD) coronary artery, which extends from left atrium to left ventricle, is the most common cause of late cardiac ischemic toxicity. An algorithm has been developed for positioning the tangential fields for homogeneous dose distribution in $\mathrm{PMO}$ and radiation dose $<10 \mathrm{~Gy}$ to the anterior descending coronary artery (14). Increasing the average dose to the heart with $1 \mathrm{~Gy}$ increases the risk of serious coronary complications by $7.4 \%$ (3). In patients with LBC, irradiation in the deep inhalation phase by controlling respiratory movements, creates the possibility of reducing the dose in the cardiac structures $(4,15)$. The expansion of the pulmonary parenchyma in deep inspirium causes a displacement of the heart and its maximum distance from the planned tumor volume (PTV) $(10,11,14)$.

The average mean heart dose was reduced from 3.7 to $1.7 \mathrm{~Gy}$ and the number of patients with $5 \%$ heart volume receiving $25 \mathrm{~Gy}$ or more was reduced from four to one of the 17 patients. The average mean LAD dose was reduced from 18.1 to $6.4 \mathrm{~Gy}$ (16) The retrospective linear regression analysis confirms, that the breathing retention technique allows a reduction in the mean heart dose without compromising homogeneous dose distribution in PTV. Regardless of the technique (DIBH or FB), mean LAD and mean ventricule dose values are significantly affected by the mean heart dose, which is halved when respiratory arrest is held in deep inspiration. Our dosimetric correlation analysis of DIBH radiation technique shows correlation between the values of mean heart dose and maximum LAD dose $/ \mathrm{R}^{2}$ $=0.5362, \mathrm{r}=0.349$. All centres showed increased V95\% target coverage and similar or lower mean heart dose (MHD) when using respiratory gating (RG) not dependent on the system being used compared to free-breathing (FB) (17). For 3D conformal RT, comparative analysis between the IMRT and 3D conformal RT during DIBH, determined significantly lower mean heart dose and contralateral breast dose $(\mathrm{p}$ $<0.01)$ at a significantly higher average dose to PTV $(p<0,01)(18)$. Regardless of the technique used (DIBH or FB), mean LAD dose and mean left ventricle dose are significantly affected by the mean heart dose. The linear regression statistical analysis reported a significant decrease in the MHD in the DIBH technique. The left breast cancer RT with the DIBH technique, significantly reduces the mean heart dose and allows controlling the maximum LAD dose and the maximum ventricle dose. The reduction in the mean cardiac dose by $3.4 \mathrm{~Gy}$ was equivalent to a $13.6 \%$ reduction in the predicted increased risk of heart disease after RT (13). The DIBH technique allows achieving lower doses to cardiac structures without significantly changing the dose homogeneity in PTV and allows for the application of more modern radiotherapy techniques $(4,15,18)$.

\section{Conclusion}

The presented dosimetric retrospective analysis in left breast cancer RT confirms the advantage of the radiation technique in the deep breathing phase. We report good control of the mean heart dose and its structures with an acceptable dose homogeneity in PTV. Regardless of the technique used (DIBH or FB), mean LAD dose and mean left ventricle dose are significantly affected by the mean heart dose. The linear regression statistical analysis reported a significant decrease in the MHD in the DIBH technique. The left breast cancer RT with the DIBH technique, significantly reduces the mean heart dose and allows controlling the maximum LAD dose and the maximum ventricle dose.

\section{References}

1. A. Jemal, F. Bray, M.M. Center, J. Ferlay, E. Ward, D. Forman Global cancer statistics. CA Cancer J Clin, 61 (2011), pp. 69-90.

2. Latest global cancer data: Cancer burden rises to 18.1 million new cases and 9.6 million cancer deaths in 2018 - PRESS RELEASE $\mathrm{N}^{\circ} 263$ - World health organization

3. Darby, S., Ewertz, M., McGale, P. et al. Risk of Ischemic Heart Disease in Women after Radiotherapy for Breast Cancer. New Engl. J. Med. 2013, 368, 987-998.

4. Latty D, Stuart KE, Wang W, Ahern V. Review of deep inspiration breath-hold techniques for the treatment of breast cancer. J Med Radiat Sci. 2015 Mar; 62(1): 74-81.

5. Darapu, A., Balakrishnan, R., Sebastian, P. et al. Is the Deep Inspiration Breath-Hold Technique Superior to the Free Breathing Technique in Cardiac and Lung Sparing while Treating both Left-Sided Post-Mastectomy Chest Wall and Supraclavicular Regions? Case Rep. Oncol. 2017, 10, 37-51.

6. Nissen HN, Appelt A. Improved heart, lung and target dose with deep inspiration breath hold in a large clinical series of breast cancer patients. Radiother Oncol. 2013;106:28-32.

7. Korreman SS, Pedersen AN, Nøttrup TJ, et al. Breathing adapted radiotherapy for breast cancer: Comparison of free breathing gating with the breath-hold technique. Radiother Oncol. 2005; 76:311-318.

8. Rice L, Goldsmith C, Green MLM, et al. An effective deepinspiration breath-hold radiotherapy technique for left-breast cancer: impact of post-mastectomy treatment, nodal coverage, and dose schedule on organs at risk. BCTT. 2017;9:437-446.

9. Damkjaer SM, Aznar MC, Pedersen AN, et al. Reduced lung dose and improved inspiration level reproducibility in visually guided DIBH compared to audio coached EIG radiotherapy for breast cancer patients. Acta Oncol. 2013;52:1458-1463.

10. Bruzzaniti, V., Abate, A., Pinnaro, P. et al. Dosimetric and clinical advantages of deep inspiration breath-hold (DIBH) during radiotherapy of breast cancer. J. Exp. Clin. Cancer Res. 2013, 32, 1-7. 
11. Parasuramar, A., DeSmit, A., Borg, M. Deep Inspiration Breath Hold Techniques in the Radiotherapeutic Management of Left-Sided Breast Cancer-Active Breathing Coordinator vs. Voluntary Breath Hold. Int. J. Gynecol. Clin. Pract. 2017, 4, 1-7.

12. Michael Ewer and Edwart Yeh. Cancer and the Heart. 2016; $p$ 90.

13. Keyvan Jabbari, Nazli Azarmahd, Shadi Babazade, Alireza Amouheidari. Optimizing of the tangential technique and supraclavicular fields in 3Dimensional Conformal Radiation Therapy for breast cancer. Journal of Medical Signals \& Sensors 2013; Vol.3; Issue 2; 107-115.

14. Cooper BT, Li X, Shin SM, et al. Pre-planning Prediction of the Left Anterior Descending Artery Maximum Dose based on Patient, Dosimetric, and Treatment Planning Parameters, Advances in Radiation Oncology (2016).

15. Smyth LM, Knight KA, Aarons YK, Wasiak J. The cardiac dosesparing benefits of deep inspiration breath-hold in left breast irradiation: A systematic review. J Med Radiat Sci 2015 Mar; 62(1):66-73.
16. Johan Vikstrom, Mari H. B. Hjelstuen, Ingvil MJaaland et al. Cardiac and pulmonary dose reduction for tangentially irradiated breast cancer, utilizing deep inspiration breath-hold with audio-visual guidance, without compromising target coverage. Acta Oncologica, 2011; 50: 42-50

17. Martin Berg, Ebbe L. Lorenzen, Ingelise Jensen, et al. The potential benefits from respiratory gating for breast cancer patients regarding target coverage and dose to organs at risk when applying strict dose limits to the heart: results from the DBCG HYPO trial, Acta Oncologica 2018, 57:1, 113-119.

18. D. M. Trifiletti, K. Wijesooriya, G. Moyer, D. Lain . Intensitymodulated radiotherapy versus three-dimensional conformal radiotherapy during deep inspiratory breath hold for left-sided whole-breast irradiation: a comparative analysis.Journal of Radiotherapy in Practice. Volume 15, Issue;1 March 2016, pp. 99-106. 